\title{
EFFECT OF KINESIOTAPE THERAPY ON CHONDROMALACIA PATELLAE
}

\author{
Moneerah Mulaih Atallah ALSHAMMERI ${ }^{1 *}$, Ebtihal Mohammed Shaim Mohammed Alshamary ${ }^{2}$, Ashwag \\ Mohammed Jarallah Alanz ${ }^{3}$, DR: Mohamed Raffat ${ }^{4}$ \\ ${ }^{* 1,2,3,4}$ College of Applied Medical Sciences Department of Physical Therapy University of Hail, KSA
}

*Corresponding Author: -

\begin{abstract}
: -
Background: Patellofemoral pain syndrome (PFPS) or chondromalacia patella is a degeneration of the patellar cartilage existing between both bone surfaces of the femur and the patella. Its most important clinical symptom is characterized by anterior knee pain that is magnified when movements from flexion to extension are performed by the knee while the patella does not move smoothly within the trochlear groove. There are several reasons that can cause this syndrome: a misalignment femoro-tibial, muscle weakness, patellar prior traumatic injury (fracture, dislocation), overuse of the joint, arthritis, etc.

Objective: Thus, the purpose of the present study is to determine if the application of KT can improve clinical outcomes, including pain and knee extensor strength and endurance, in physically active patients diagnosed with PFPS.

Methodology: After a verbal explanation of the study protocol, the selected 8 participants were randomly divided into two groups including control group $A(n=3)$ and treatment group $B(n=5)$. Each participant in group $B$ received Kinesio tape $(K T)$ for four days without any special exercises (Fig. 3). While participants in group A, were not treated with any other treatments but were taught with health education concerning with PFPS.

Results: Results showed an improvement across group B in pain and disability scores, as illustrated in Tables $1 \& 2$ and Figure 3. In group A, the average VAS was $6.5 \mathrm{~cm}, 6.3 \mathrm{~cm}, 5.7 \mathrm{~cm}$, and $5.4 \mathrm{~cm}$, respectively from the first to the fourth day. While results of treated group B showed that the average VAS was $6.7 \mathrm{~cm}, 5.8 \mathrm{~cm}, 4.7 \mathrm{~cm}$, and $3.02 \mathrm{~cm}$, respectively from the first to the fourth day.

Conclusion: Based on the current study, Kinesio taping is significantly improve muscle activity, motor function, and quality of life, benefits which are possibly facilitated by pain relief.

However, further research examining the mechanism of pain relief is required.
\end{abstract}

Key wards: Patellofemoral, pain, Kinesio tape.

\section{(우 $($ (1)}




\section{INTRODUCTION}

Patellofemoral pain syndrome (PFPS) or chondromalacia patella is a degeneration of the patellar cartilage existing between both bone surfaces of the femur and the patella. Its most important clinical symptom is characterized by anterior knee pain that is magnified when movements from flexion to extension are performed by the knee while the patella does not move smoothly within the trochlear groove. There are several reasons that can cause this syndrome: a disalignment femoro-tibial, muscle weakness, patellar prior traumatic injury (fracture, dislocation), overuse of the joint, arthritis, etc. The main objectives to rehabilitate this pathology will be to reduce the pain and improve the neuropropioceptive information.

In the patellofemoral joint, the patella serves as a link to converge the fibers of the quadriceps femoris muscle group to increase its lever arm and maximize its mechanical advantage. To ensure this functional efficacy, maintaining the patellar alignment in the trochlear groove of the femur is necessary. Malalignment of the patella, or altered patellar tracking, may be a predisposing factor for patellofemoral pain, chondromalacia, and articular cartilage degeneration.

Numerous biomechanical characteristics of the lower body such as decreased joint flexibility, large quadriceps angle (Qangle), and increased subtalar joint pronation have been proposed to explain its onset. Nonetheless, the leading etiological factor is a decline in the activation of the vastus medialis oblique (VMO) muscle. Anatomically, the VMO attaches at a $40-55^{\circ}$ angle to the long axis of the femur on the medial side of the patella. The functional importance of the $\mathrm{VMO}$, therefore, is to dynamically stabilize the patella on the medial side and prevent lateral deviation and rotation of the patella caused by the lateral pull of the larger vastus lateralis (VL) muscle.

Patellofemoral pain syndrome (PFPS) is a clinical condition that is characterized by retropatellar and/or peripatellar pain associated with activities involving lower limb loading (eg, walking, running, jumping, stair climbing, and prolonged sitting and kneeling). PFPS is the most common overuse injury of the lower extremity, and is particularly prevalent in those who are physically active. For example, approximately 2.5 million runners will be diagnosed with PFPS in a given year. PFPS also is a significant problem in the military as it has been reported that $37 \%$ of recruits develop PFPS while in basic combat training. Females are reported to be at greater risks for PFPS than their male counterparts. Alarmingly, 70\% to $90 \%$ of individuals with PFPS have recurrent or chronic pain. In addition, the result of a recent study suggest that having PFPS as a younger individual may predispose one to develop patellofemoral osteoarthritis later in life.

Patellofemoral pain syndrome also may result from the way your hips, legs, knees, or feet are aligned. This alignment problem can be caused by your having wide hips or underdeveloped thigh muscles, being knock-kneed, or having feet with arches that collapse when walking or running (a condition called over-pronation).

The main symptom is pain behind the kneecap. You may have pain when you walk, run, or sit for a long time. The pain is generally worse when walking downhill or down stairs. Your knee may swell at times. You may feel or hear snapping, popping, or grinding in the knee. Patellofemoral pain syndrome can best be prevented by strengthening your thigh muscles, particularly the inside part of this muscle group. It is also important to wear shoes that fit well and that have good arch supports.

Several factors may be associated with patellar malalignment. An increase in Q angle (more than $15^{\circ}$ ) may increase the lateral pull of the patella, causing the patella to glide on the lateral ridge of the femoral groove and producing pain. Tightness of the muscles that cross the knee joint may have an effect on patellar alignment. A tight rectus femoris muscle can limit patellar movement, reducing the functional and mechanical efficacy of the patellofemoral joint. A tight iliotibial band may pull the patella laterally during knee flexion, whereas tightness in the hamstring muscle group may increase patellofemoral joint reaction forces because of an increased knee flexion moment. Gastrocnemius tightness may limit ankle dorsiflexion, which can result in increased subtalar joint pronation and tibial internal rotation, contributing to abnormal pulling of the patellar tendon and the patellar malalignment.

The effectiveness of patellar taping on the VMO activity of individuals suffering from PFPS is unclear. Support for the use of patellar taping to alter the activity of the VMO muscle comes from either not well-controlled studies using single-joint tasks or directly from McConnell's research group. To date, no study has systematically examined the effects of taping the patella in various directions on the magnitude of VMO activity and perceived pain, especially during a velocity controlled multi-joint movement.

McConnell taping is a frequently utilized patellar taping technique that is proposed to have up to three effects: patellar glide, patellar tilt, and patellar rotation. The McConnell medial glide technique is commonly used in the treatment of PFPS. In these patients, the femur tends to adduct relative to a stable patella during movement. By applying a medial glide McConnell taping, it is hypothesized patellar tracking will be improved due to the force of the tape pulling the patella medially and improving patellofemoral kinematics.

Kinesio tape (KT) is a relatively new modality that has become popular with athletes and clinicians alike in the treatment of musculoskeletal pathologies. It is a cotton and elastic blend athletic tape that was designed to match the texture and elasticity of human skin. It is theorized that the use of KT can enhance joint stability by providing support to or around affected muscles. Additionally, it has been proposed that KT can be used for neuromuscular reeducation, for pain reduction, to improve performance, to prevent injury, and to improve circulation and healing. These claims purport that KT is useful in both injured and uninjured populations; however, the physiological basis for these claims are not yet well understood. It is possible that KT may function to reduce pain in PFPS patients by providing them with cutaneous stimulation via large afferent fibers achieved through deformation of the slowly adapting mechanoreceptors in the skin. It is also proposed that KT may facilitate muscle contraction, the mechanism for which remains unknown.

While KT application has become increasingly popular in recent years, evidence to support its efficacy has been conflicting. In healthy individuals, the application of KT at varying sites, including the forearm, shank, and thigh, has not been shown to improve strength, endurance, or functional performance. In injured individuals, the efficacy of KT 
application is conflicting. In two separate systematic reviews of the use of KT to treat pathologies, Morris et al. and Mostafavifar et al. concluded that more research needs to be done before KT can be recommended for clinical use over other treatments. The latter indicated that the benefits with regard to pain could not be entirely discounted. Both reviews also noted a lack of high-quality randomized controlled trials (RCTs) available for systematic review. Conversely, other nonRCT research has shown that KT has beneficial effects on both pain and functional performance in patients with shoulder impingement and lateral ankle sprain.

Thus, the purpose of the present study is to determine if the application of KT can improve clinical outcomes, including pain and knee extensor strength and endurance, in physically active patients diagnosed with PFPS.

\section{Methods}

Participants enrolled in this study must have been diagnosed with PFPS. The participants with PFPS presented with diffuse, unilateral anterior knee pain for at least 8 weeks, exacerbated by stair climbing, sitting, walking, running, squatting, knee flexion, and isometric quadriceps contraction, and had never received patellar taping before this study. None of the patients with PFPS were participating in rehabilitation at the time of the study.

\section{Inclusion criteria}

Eight participants (4 males and 4 females) with PFPS (age from 23 to 51years, height from 158 to $181 \mathrm{~cm}$, mass from 49 to $102 \mathrm{~kg}$ ) volunteered for this study. All participants were recruited from Physical Therapy Department, King Khalid Hospital at Hail, Saudi Arabia. After they agreed to participate in the study, they receiving explanations regarding the purpose and procedures of the study, and signed an informed consent statement before participation. At the time of the study the participants were not receiving any medical or physical therapy.

After a verbal explanation of the study protocol, the selected 8 participants were randomly divided into two groups including control group A $(n=3)$ and treatment group $\mathrm{B}(n=5)$. Each participant in group $\mathrm{B}$ received Kinesio tape (KT) for four days without any special exercises (Fig. 3). While participants in group A, were not treated with any other treatments but were taught with health education concerning with PFPS (figs. $1 \& 2$ ).

\section{Exclusion criteria}

Participants were excluded if they were previously diagnosed with osteoarthritis or patellar tendinitis or tendinopathy; had a previous history of surgery (including arthroscopy), fracture, patellar dislocation/subluxation, or ligamentous or other soft tissue injury; or had a concussion within the last year or suffered from any neurologic deficit.

\section{Outcome measures}

The outcome measures used in this study were the Oswestry Disability Questionnaire (ODQ) and the Visual Analogue Scale (VAS) both of which were scored by all participants after four-weeks.

\section{Data analysis}

Standard deviation and mean for the obtained data were calculated. Also, the data obtained were analyzed by SPSS 13.0 software test to compare the data before and after treatment within groups. The significant threshold set at $p<0.05$, or non-significant set at $p>0.05$.

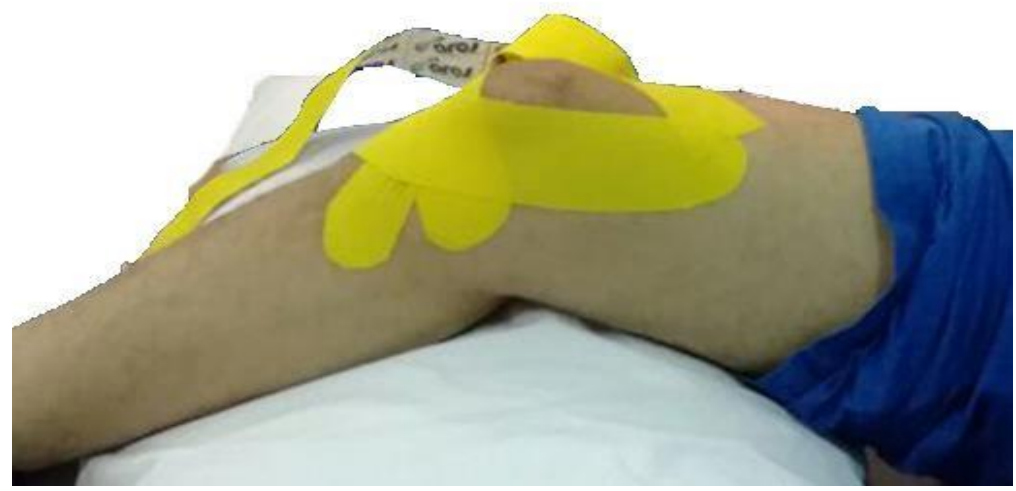

Fig. 1: Showing a male participant during received Kinesio tape (KT). 


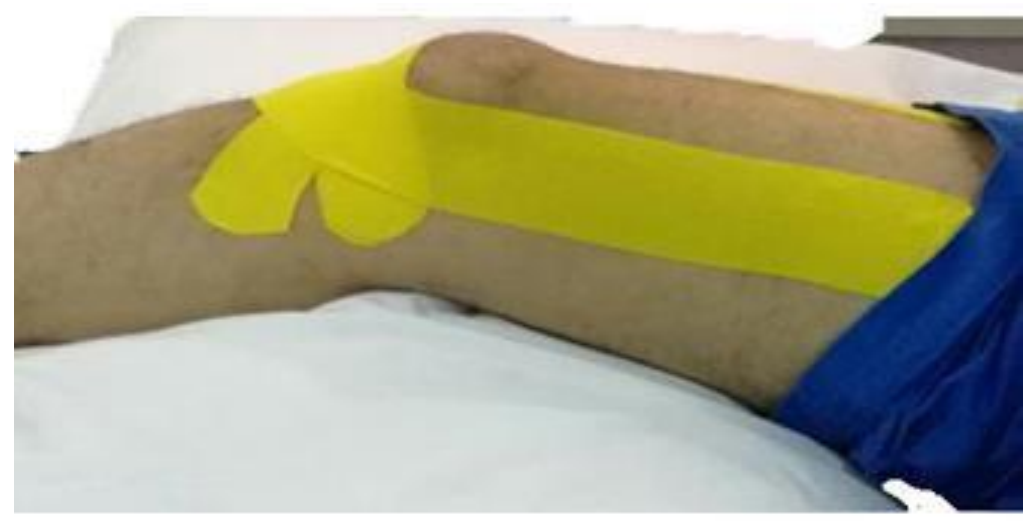

Fig. 2: Showing a male participant after received Kinesio tape (KT).

\section{Results}

The VAS scale is a valid and reliable tool to rate pain intensities along a $10 \mathrm{~cm}$ line. The patient is asked to put a mark along this line to reflect the intensity of the pain.

A total of 8 participants were eligible to take part in this study. These were divided into 2 groups; control group A $(n=3)$ and treated group B $(n=5)$. The recorded data, as measured by the standardized visual analog scale VAS, showed a statistically significant difference among treated and control groups.

Results showed an improvement across group B in pain and disability scores, as illustrated in Tables $1 \& 2$ and Figure 3. In group A, the average VAS was $6.5 \mathrm{~cm}, 6.3 \mathrm{~cm}, 5.7 \mathrm{~cm}$, and $5.4 \mathrm{~cm}$, respectively from the first to the fourth day. While results of treated group B showed that the average VAS was $6.7 \mathrm{~cm}, 5.8 \mathrm{~cm}, 4.7 \mathrm{~cm}$, and $3.02 \mathrm{~cm}$, respectively from the first to the fourth day.

As compared the obtained results of the two groups; control and treated we can analyzed the following; at the end of the first day there was no a statistically significant difference among treated and control groups, where the values of two groups was 6.5 for A and 6.7 for B. Also, at the end of the second day, the results showed no a statistically significant difference among treated and control groups. However, at the end of third and fourth day, the results showed a statistically significant difference among treated and control groups; where, the VAS for A group was 5.7 and 5.4 for $3^{\text {rd }}$ and $4^{\text {th }}$ day, but in treated group B values of VAS was 4.7 and 3.02 for the $3^{\text {rd }}$ and $4^{\text {th }}$ day.

From the result mentioned above, the kinesio tape effectiveness was time dependent and the perfect role of it needs a short period of time to appear. Also, it is more effective protocol in treatment of patellofemoral pain.

Table 1: Showing the values of VAS along the period of the study.

\begin{tabular}{||c||c|c|c|c||c|c|c|c|}
\hline & \multicolumn{3}{|c|}{ Control group A } & \multicolumn{3}{|c|}{ Treated group B } \\
\hline \hline VAS $(\mathbf{c m})$ & $1^{\text {st }}$ day & $2^{\text {nd }}$ day & $3^{\text {rd }}$ day & $4^{\text {th }}$ day & $1^{\text {st }}$ day & $2^{\text {nd }}$ day & $3^{\text {rd }}$ day & $4^{\text {th }}$ day \\
\cline { 2 - 9 } & 6.5 & 6.3 & 5.7 & 5.4 & 6.7 & 5.8 & 4.7 & 3.02 \\
\hline
\end{tabular}

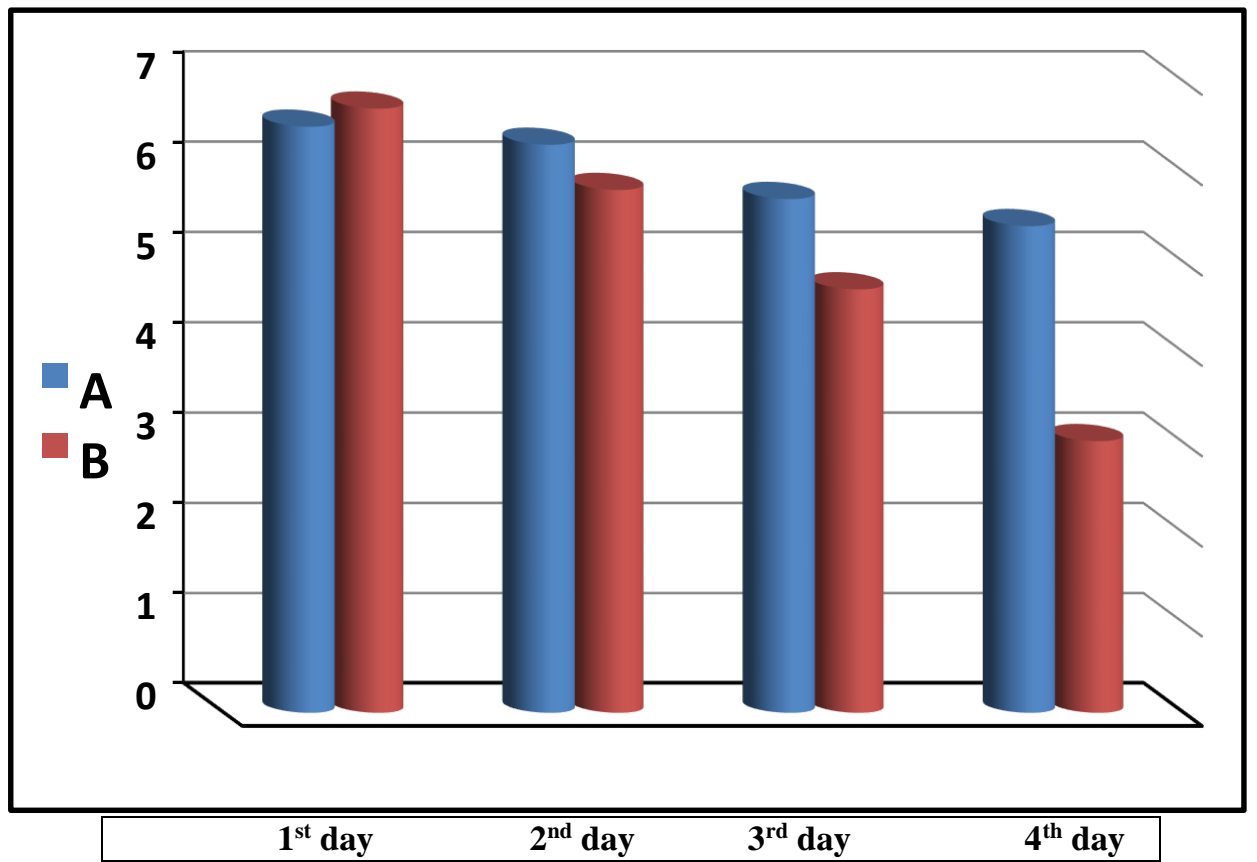

Fig. 3: Showing a histogram for the obtained values of VAS. 
Table 2: Comparison of the mean pain density between the different stages in the group $B$.

\begin{tabular}{|c|c|c|c|c|}
\hline & $\mathbf{1}^{\mathrm{ST}}$ day & $2^{\text {nd }}$ day & $3^{\text {rd }}$ day & $4^{\text {th }}$ day \\
\hline $\begin{array}{l}\text { oup B Min. - } \\
\text { Max. }\end{array}$ & $11.50-43.0$ & $17.3-51.0$ & $33.0-61.0$ & $38.0-98.0$ \\
\hline Mean \pm SD & $18.38 \pm 2.15$ & $23.41 \pm 3.47$ & $82.0 \pm 4.97$ & $416.75 \pm 3.0$ \\
\hline Median & 19.50 & 23.5 & 71.0 & 410.50 \\
\hline $\mathbf{p}_{1}$ & & & 0.359 & $<0.001^{*}$ \\
\hline $\mathbf{p}_{2}$ & & & \multicolumn{2}{|c|}{$0.010^{*}$} \\
\hline
\end{tabular}

\section{p1: Stands for adjusted LSD p-value for ANOVA with repeated measures for comparison between one month with each other period \\ p2: Stands for adjusted LSD p-value for ANOVA with repeated measures for comparison between 1.5 month with two months \\ *: Statistically significant at $\mathbf{p} \leq \mathbf{0 . 0 5}$}

\section{Discussion}

Kinesio taping has favorable properties and is composed of waterproof and ventilative material. Patients like Kinesio taping because its favorable adhesive properties facilitate easy use and prevent allergic reactions; thus, Kinesio taping is in widespread clinical use. Kinesio taping involves affixing Kinesio tape to the skin folds to increase the space between the muscle and fascia. Kinesio tape can increase local blood or lymphatic circulation.

There are two approaches to Kinesio taping. First, the tape can be applied in the direction of muscle contraction from muscle origin to the insertion; this method facilitates the contraction of injured muscles. The second approach involves adhering the tape in the opposite direction of muscle contraction from the muscle insertion to origin. It prevents muscle overuse to provide excessive muscle tension. McConnell taping uses a different approach and material for affixing the rigid tape to correct patellar alignment. Three components of patellar orientation, such as glide component, tilt component, and rotation component, were assessed before the patellar tape.

The principle of the taping is to restrict abnormal patella tracking, thus reducing joint friction and oppression of the injured tissue. However, Kinesio taping uses elastic tape to provide active muscle contraction and increase the pull tension of the patella. Therefore, the materials, uses, and principles of the two patellar taping approaches differ. Lim et al. indicated that the elastic material of Kinesio tape is similar to the skin and soft tissue and its elasticity allows it to elongate to $130 \%$ to $140 \%$ of its initial length. When Kinesio tape is applied with different methods on muscle, Kinesio taping provides tension force to pull or inhibit muscle contraction. The skin folds caused by tape elasticity can increase local lymph and blood circulation, and metabolic substances can be removed. These factors may be possible causes of the analgesic effect.

Kinesio tape consists of thin, cotton, and acrylic-acid containing porous fabric. The tape is a unique and non-latex adhesive patch. Akbas, et al. applied Kinesio taping to PFPS patients by adhering Kinesio tape onto the VMO muscle to increase proprioception input and to facilitate muscle contraction. In addition, they applied the tape over the iliotibial band, tensor fasciae latae, and VL muscle to inhibit muscle tension and relax the muscle. Lan et al. used Kinesio taping to imitate the use of McConnell taping. They used the hands to push the patella to the inside and applied Kinesio tape to fix the patella. Subsequently, a PFPS patient repeatedly stepped up and down on an 8-inch step and induced pain was assessed.The results revealed that Kinesio taping reduces PFPS-associated pain but does not change patellar alignment. Gonz'alez-Iglesias et al. believe that Kinesio taping can be applied at 50\% to $85 \%$ tensions on the skin to restrict partial or full joint motion, but the taping tension was insufficient to correct the patellar alignment.

Kinesio taping pulls the skin to increase the gap between the skin and muscle, reduces tissue edema, and promotes blood and lymphatic circulation; thus, Kinesio taping effectively relieves pain. Positive effects of the taping were also seen on reduced swelling and muscle spasms of PFPS patients. Several studies suggest that Kinesio taping is useful in treating acute sports injuries because it immediately reduces pain, improves muscle contracture, and accelerates athletes' return to normal activity. However, studies proving the effects of Kinesio taping are still rare, and their results are inconsistent.

The results of several studies indicate that Kinesio and McConnell taping can reduce pain in PFPS patients. The mechanism of Kinesio taping that affects pain in patients with PFPS remains unclear, and evidence of the underlying mechanism by which McConnell taping affects patients with PFPS is also weak. Most studies consistently indicate that Kinesio taping or McConnell taping can stimulate cutaneousmechanoreceptors and improve knee proprioception. 5.

\section{Conclusion}

Kinesio taping is a type of patellar taping used to treat patients with PFPS. Kinesio taping can be applied to muscles to relieve pain, but there is a lack of evidence on effect of the effects of taping on patellar alignment correction. Based on the current study, Kinesio taping is significantly improve muscle activity, motor function, and quality of life, benefits which are possibly facilitated by pain relief. However, further research examining the mechanism of pain relief is required. 


\section{References}

[1].Christou EA. Patellar taping increases vastus medialis oblique activity in the presence of patellofemoral pain. $\mathbf{J}$ Electromyogr Kinesiol. 2004;14: 495-504.

[2].Ng GYF, Cheng JMF. The effects of patellar taping on pain and neuromuscular performance in subjects with patellofemoral pain syndrome. Clin Rehabil. 2002;16:821-827.

[3].McConnell J. The management of chondromalacia patellae: a long term solution. Aust J Physiother. 1986;32:215223.

[4].Baker MM, Juhn MS. Patellofemoral pain syndrome in the female athlete. Clin Sports Med. 2000;19:315-329.

[5].Nyland JA, Ullery LR, Caborn DNM. Medial patellar taping changes the peak plantar force location and timing of female basketball players. Gait Posture. 2002;15:146-152.

[6].Ireland ML, Willson JD, Ballantyne BT, Davis IM. Hip strength in females with and without patellofemoral pain. J Orthop Sports Phys Ther. 2003;33:671-676.

[7].Brindle TJ, Mattacola C, McCrory J. Electromyographic changes in the gluteus medius during stair ascent and descent in subjects with anterior knee pain. Knee Surg Sports Traumatol Arthrosc. 2003;11:244-251.

[8].Holmes SW Jr, Clancy WG Jr. Clinical classification of patellofemoral pain and dysfunction. J Orthop Sports Phys Ther. 1998;28:299-305.

[9].Gilleard W, McConnell J, Parsons D. The effect of patellar taping on the onset of vastus medialis obliquus and vastus lateralis muscle activity in persons with patellofemoral pain. Phys Ther. 1998;78:25-32.

[10]. Bockrath K, Wooden C, Worrell T, Ingersoll CD, Farr J. Effects of patellar taping on patella position and perceived pain. Med Sci Sports Exerc. 1993;25:989-992.

[11]. Baker V, Bennell K, Stillman B, Cowan S, Crossley K. Abnormal knee joint position sense in individuals with patellofemoral pain syndrome. J Orthop Res. 2002;20:208-214. Brukner P, Khan K, eds. Clinical Sports Medicine. 2nd ed. Roseville, NSW, Australia: The McGraw-Hill Book Co Australia Pty Ltd; 2001.

[12]. Clark DI, Downing N, Mitchell J, Coulson L, Syzpryt EP, Doherty M. Physiotherapy for anterior knee pain: a randomised controlled trial. Ann Rheum Dis. 2000;59:700-704.

[13]. Ernst GP, Kawaguchi J, Saliba E. Effect of patellar taping on knee kinetics of patients with patellofemoral pain syndrome. J Orthop Sports Phys Ther. 1999;29:661-667.

[14]. Osorio J.A., Vairo J.L., Rozea G.D., Bosha P.J., Millard R.L., Aukerman D.F., Sebastianelli W.J. The effects of two therapeutic patellofemoral taping techniques on strength, endurance and pain responses. Physical Therapy in Sport. 2013; 14 (4): 199-206.

[15]. Osterhues D. The use of KT in the management of traumatic patella dislocation: a case study. Physiotherapy Theory and Practice. 2004; 20: 267-270.

[16]. Quilty B., Tucker M., Campbell R., Dieppe P. Physiotherapy, including quadriceps exercises and patellar taping, for knee osteoarthritis with predominant patello-femoral joint involvement: randomized controlled trial. Journal of Rheumatology. 2003; 30 (6): 1311- 1317.

[17]. Smith T., McNamara I., Donell S. The contemporary management of anterior knee pain and patellofemoral instability. The Knee. 2013; 20 (S1): 3-15.

[18]. Yang L., Jiang J.L, Liang Q., Lei Z.J., He C.Q. The effect of elastic taping on patients with patellofemoral syndrome. Journal of Sichuan University. Medical science edition. 2014; 45 (1): 126-128.

[19]. Powers, C. M. (1998). Rehabilitation of patellofemoral joint disorders: a critical review. Journal of Orthopaedic \& Sports Physical Therapy, 28, 345e354.

[20]. Puniello, M. S. (1993). Iliotibial band tightness and medial patellar glide in patients with patellofemoral dysfunction. Journal of Orthopaedic \& Sports Physical Therapy, 17, 144e148.

[21]. Riemann, L. (2002). The sensorimotor system, part I: the physiologic basis of functional joint stability. Journal of Athletic Training, 71e79.

[22]. Salsich, G. B., Brechter, J. H., Farwell, D., \& Powers, C. M. (2002). The effects of patellar taping on knee kinetics, kinematics, and vastus lateralis muscle activity during stair ambulation in individuals with patellofemoral pain. Journal of Orthopaedic \& Sports Physical Therapy, 32, 3e10.

[23]. Tunay, V., Akyuz, A., Onal, S., Usgu, G., Dogan, G., Teker, B., et al. (2008). Comparison of the instant effects of kinesio and McConnell patellar taping on performance in patellofemoral pain syndrome. Fizyoterapi Rehabilitasyon, 19(3), 104e109. 